\title{
Avaliação cintilográfica de diferentes dosagens de Tecnécio-99m na padronização da perfusão pulmonar em cães da raça Rottweiller
}

\author{
Dosage padronization of ${ }^{99 m}$ TC for scintigraphy evaluation on the pulmonary perfusion in \\ Rottweiller dog
}

\author{
Gabriela Silva Rodrigues ${ }^{I}$ Ricardo Romão Guerra ${ }^{\mathrm{II}}$ Flávio Ribeiro Alves ${ }^{\mathrm{III}}$ \\ André Luís Rezende Franciolli ${ }^{\mathrm{IV}}$ Phelipe Oliveira Favaron ${ }^{\mathrm{IV}}$ Fabio Luiz Navarro Marques ${ }^{\mathrm{V}}$ \\ Patricia Russano Cuyumjian $^{\mathrm{VI}}$ Maria Angélica Miglino ${ }^{\mathrm{IV}}$ Horácio Luis Tommasi Junior $^{\mathrm{IV}}$ \\ Carlos Alberto Buchpiguel ${ }^{V}$ Pedro Primo Bombonato ${ }^{\mathrm{IV}}$
}

\section{RESUMO}

A avaliação da função pulmonar em cães pode ser obtida mediante exames cintilográficos, os quais incluem a injeção de radiofármacos, seguida de radiografias. Neste estudo, foram utilizados 10 cães machos hígidos da raça Rottweiler e radiograficamente normais e soronegativos para dirofilariose, com administração de doses variadas de macroagregado de albumina sérica humana marcados com tecnécio-99m ([ $\left.\left.{ }^{99 m} T c\right](M A A)\right)$. Os resultados foram avaliados qualitativa e quantitativamente, considerando o índice de perfusão pulmonar e sua homogeneidade, sendo dessa maneira visualizado um aumento na homogeneidade da imagem diretamente relacionado ao número de partículas de MAA injetado. Este estudo define os critérios de normalidade e de dosagem na perfusão pulmonar em cães hígidos da raça rottweiller estabelecidos com a técnica de cintilografia.

Palavras-chave: cintilografia pulmonar, cães, medicina nuclear, Tecnécio-99m.

\section{ABSTRACT}

Pulmonary function evaluation in dogs can be gotten by scintigraphy exams, which are obtained with radiopharmacs injections, followed by radiography. In this study it was used 10 healthful male dogs of Rottweiler breed, radiographically normal and serum negative for dirofilariasis, with administration of varied doses of human serum albumin grouped marked with technetium-99m (MAA). The results were evaluated qualitatively and quantitatively, considering the index of pulmonary perfusion and its homogeneity, being found an increase on the homogeneity of image directly related with the number of MAA particles injected. This study defines the criteria of normality and the dosage of pulmonary perfusion in healthful dogs established with scintigraphy technique.

Key words: pulmonary scintigraphy, dogs, nuclear medicine, Technetium-99m.

\section{INTRODUÇÃO}

O diagnóstico por imagem da maioria das doenças pulmonares baseia-se essencialmente no reconhecimento de alterações morfológicas e funcionais. Tais anormalidades apresentam-se mais comumente como variações no tamanho, na forma, na margem, na opacidade, na textura ou na atividade do tecido pulmonar, dependendo da técnica de imagem empregada (TIDWELL, 1992).

Na radiologia, características de imagens são atribuídas a alterações que afetam particularmente as estruturas anatômicas dos campos pulmonares, como o interstício, os alvéolos, os brônquios e os vasos. Contudo, em alguns casos, o uso do exame radiográfico parece ser limitado, como, por exemplo, nos animais que manifestam sintomatologia de doença pulmonar e apresentam imagem radiográfica torácica normal. A

${ }^{\mathrm{I}}$ Médica Veterinária Autônoma, Cotias, SP, Brasil.

${ }^{\mathrm{II} C e n t r o ~ d e ~ C i e ̂ n c i a s ~ A g r a ́ r i a s, ~ U n i v e r s i d a d e ~ F e d e r a l ~ d a ~ P a r a i ́ b a ~(U F P B), ~ 58397-000, ~ A r e i a s, ~ P B, ~ B r a s i l . ~ E-m a i l: ~ r i c a r d o @ c c a . u f p b . b r . ~}$ Autor para correspondência

IIICurso de Medicina Veterinária, Universidade Federal do Piauí (UFPI), Campus Cinobelina Elvas, Bom Jesus, PI, Brasil.

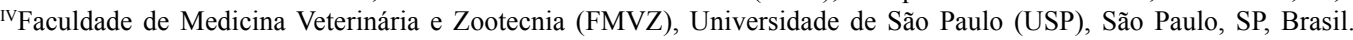

'Faculdade de Medicina, USP, São Paulo, SP, Brasil.

${ }^{\mathrm{VI} M e ́ d i c a}$ Veterinária Autônoma, São Paulo, SP, Brasil. 
afecção pulmonar pode ser inaparente em radiografias, cujas alterações morfológicas sejam insuficientes para comprometerem a radiopacidade normal dos pulmões (TIDWELL, 1992).

A aplicação das técnicas de medicina nuclear, estudando a forma e a função do órgão, vem evoluindo ultimamente (CANECA et al., 2002). A cintilografia aparece como uma técnica alternativa, sensível e minimamente invasiva (BALOGH et al., 1999). Em pequenos animais, o exame cintilográfico de perfusão pulmonar é mais comumente utilizado em pacientes com suspeita de tromboembolismo, ainda que também possa apresentar alterações nos casos de vasoconstrições pulmonares e destruições do leito capilar de outras etiologias (HARNAGEL et al., 1989). Internacionalmente, o exame cintilográfico já se encontra consolidado na rotina médico-veterinária, como forma de caracterizar as mais diversas alterações, dentre elas, as lesões cardíacas, ósseas e pulmonares (SUGERMAN et al., 1981; SARIERLER et al., 2005; SCHWARZ et al., 2004; DANIEL et al., 2008). No Brasil, diversos profissionais têm se dedicado à pesquisa, com vistas ao estabelecimento de protocolos rápidos, seguros e de baixo custo, que já se encontram disponíveis em centros de pesquisa especializados (MARTIN et al., 1995; MARTIN, 2003).

Dessa forma, este estudo propõe o uso da cintilografia, com administração de doses variadas de macroagregado de albumina sérica humana marcado com tecnécio-99m ([ ${ }^{99 m}$ Tc](MAA)), visando a padronizar essa técnica em perfusão pulmonar de cães radiograficamente normais. Além disso, o trabalho busca avaliar qualitativa e quantitativamente a obtenção das imagens no sentido de definição de critérios de normalidade, segundo essa metodologia.

\section{MATERIAL E MÉTODOS}

O grupo experimental foi constituído por 10 cães machos adultos e hígidos da raça Rottweiler, com idades variando entre dois e seis anos, e peso corpóreo de 36 a $45 \mathrm{~kg}$, provenientes 2o Batalhão da Polícia do Exército Brasileiro. Para todos os animais, foi realizado exame para detecção da Dirofilaria immitis, como critério de exclusão, a fim de se evitar a alteração do padrão vascular pulmonar dos animais estudados, conforme previamente estabelecido por THRALL et al. (1979).

As cintolografias foram realizadas no Centro de Medicina Nuclear do Departamento de Radiologia da Universidade de São Paulo, o qual conta com laboratório de radiofarmácia capacitado para produção do conjunto de reativos liofilizados para marcação com tecnécio [ ${ }^{99 \mathrm{~m}} \mathrm{TC}$ ] e com câmara de cintilação. Levando em consideração as avaliações cintilográficas realizadas por RHODES et al (1971) e BROOKSTEIN et al. (1980), foi estabelecido o protocolo ao qual cada cão foi submetido a três diferentes estudos cintilográficos com a administração de 50.000, 100.000 e 300.000 partículas de macroagregados de albumina sérica humana ${ }^{a}$, respeitando um intervalo de pelo menos cinco dias entre cada tratamento. A escolha do intervalo e de tempo das doses administradas teve como objetivo eliminar a heterogeneidade, devido ao número insuficiente de micropartículas, conforme protocolo utilizado por HECK \& DULEY (1974) em pacientes humanos.

As imagens foram obtidas por meio de câmara de cintilação modelo Orbiter ZLC $750^{\mathrm{b}}$. Os animais foram sedados pela associação de acepromazina $^{c}$ e cloridato de petidina ${ }^{\mathrm{d}}$ nas doses de $0,1 \mathrm{mg} \mathrm{kg}^{-1}$ e $2,0 \mathrm{mg} \mathrm{kg}^{-1}$, respectivamente, via intramuscular.

As análises qualitativas foram realizadas por três observadores empregando-se uma escala de cinza, referente ao padrão de distribuição normal quando observada homogeneidade na distribuição do composto nos campos pulmonares, de acordo com o seguinte sistema de escore: 0 (normal); 1 (heterogêneo difuso): a) discreto, b) moderado e c) acentuado; e 2 (defeito focal): a) um, b) de dois a quatro, c) mais de quatro.

As análises quantitativas se basearam na quantificação da perfusão pulmonar, referente às imagens em projeção dorsal e ventral, sendo realizado cálculo dos índices de perfusão totalizando seis regiões pulmonares definidas em cada projeção. O cálculo da uniformidade integral foi realizado conforme previamente descrito por PRICE et al. (1990); OCH et al. (1992) e BUCKLEY et al. (2006), tomando como base a seguinte fórmula:

U1*=100.Máximo de contagens-Mínimode contagens $=\%$ Máximo de contagens + Mínimo de contagens

$\mathrm{U} 1^{*}=$ uniformidade integral.

O teste estatístico aplicado foi a análise de variância (ANOVA) de medidas repetidas, com o uso do programa de computador GraphPad InStat ${ }^{(}$, comparando os valores da distribuição relativa do radiofármaco nas diferentes regiões pulmonares, obtidos com as doses de 50.000, 100.000 e 300.000 partículas de MAA, ao nível de significância de 5\%. Os diferentes valores obtidos com o cálculo da uniformidade integral nas imagens derivadas das três doses de MAA foram comparados por meio do mesmo teste estatístico ao nível de significância de 5\%. 


\section{RESULTADOS}

As imagens obtidas com diferentes números de partículas de $\left[{ }^{99 \mathrm{~m}} \mathrm{Tc}\right](\mathrm{MAA})$, nas projeções dorsal, ventral, lateral direita e esquerda, mantiveram as mesmas características visuais (Figura 1).

Os campos pulmonares apresentaram-se relativamente simétricos nas projeções dorsais e ventrais. Segundo a verificação dos três observadores, o pulmão direito estendeu-se de forma discreta mais caudalmente que o esquerdo em algumas imagens dorsais.

A área cardíaca foi representada por uma região circunscrita, ovalada e central, de baixa perfusão, nas projeções dorsal e ventral, sendo mais evidente nesta última e apresentando-se deslocada para a esquerda em sua porção caudal. Também pode ser evidenciada de forma tênue nas projeções laterais. As regiões craniais apareceram menos perfundidas em todas as imagens, enquanto que os campos pulmonares caudais apresentaram maior perfusão na projeção ventral, e as porções médias foram representadas por áreas de maior captação na imagem dorsal.

Uma zona aproximadamente triangular foi observada em correspondência à porção média e caudal dos pulmões, caudal à sombra da silhueta cardíaca quando nas projeções laterais.

Sob avaliação qualitativa, todas as imagens utilizadas para a realização do experimento foram enquadradas na classificação do "escore 0" pelos três

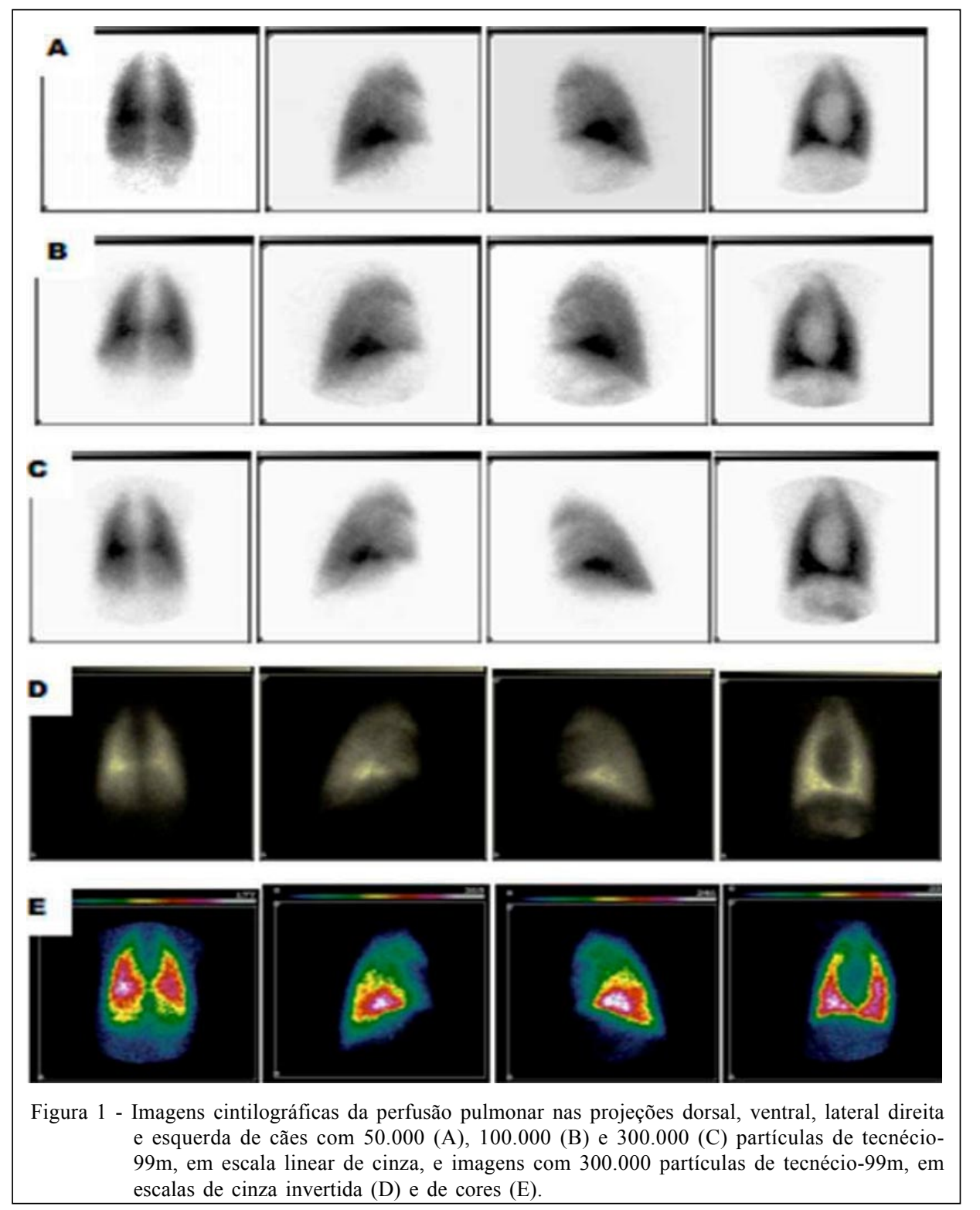

Ciência Rural, v.40, n.3, mar, 2010. 
observadores. A Análise de Variância (ANOVA) demonstrou que a distribuição do radiofármaco nas regiões pulmonares cranial, média e caudal apresentou diferença estatística $(\mathrm{P}<0,05)$, determinada pela infusão das três dosagens padronizadas para o experimento, como descritas na tabela 1. Dentro da mesma região, dosagens diferentes de infusão não demonstraram diferença significativa para nenhuma das três regiões analisadas (região cranial $\mathrm{P}=0,867$; região medial $\mathrm{P}=0,6856$; e região caudal $\mathrm{P}=0,6254$ ).

Os valores de uniformidade integral (UI) mostraram aumento de valores à medida que foi aumentada a infusão do número de partículas injetadas. Assim sendo, foi observada diferença significativa entre as médias dos valores da uniformidade integral, quando comparados os grupos 50.000 e 300.000 $\left(\mathrm{q}_{\mathrm{cal}}=8,3369, \mathrm{q}_{\mathrm{tab}}=3,509, \mathrm{P}<0,05\right)$, assim como entre os grupos 100.000 e $300.000\left(\mathrm{q}_{\mathrm{cal}}=5,951\right), \mathrm{q}_{\mathrm{tab}}=3,509$, $\mathrm{P}<0,05)$, não havendo diferença entre os grupos em que foram injetadas 50.000 e 100.000 partículas de microagregados $\left(\mathrm{q}_{\mathrm{cal}}=2,415, \mathrm{q}_{\mathrm{tab}}=3,509, \mathrm{P}>0,05\right)$, respectivamente (Tabela 2 ).

\section{DISCUSSÃO}

Segundo FLÜCKINGER \& GOMEZ (1984), quando presentes, as alterações radiográficas mais comumente observadas nos casos de embolismo pulmonar são caracterizadas por áreas de hipertransparência ou hipovascularização, onde podem predominar padrões alveolares, em decorrência de sua baixa perfusão e como conseqüência da presença de hemorragia, infarto pulmonar ou efusão pleural. Porém, sabe-se que tais padrões radiográficos não são excluídos do TEP e podem ser facilmente interpretados como alterações pulmonares de outras origens. A radiografia torácica simples é o método de auxilio ao diagnóstico mais amplamente utilizado na avaliação de afecções pulmonares dentro da Medicina Veterinária

Tabela 1 - Distribuição dos valores médios da dosagem do radiofármaco $\left(\left[{ }^{99 m} \mathrm{Tc}\right](\mathrm{MAA})\right)$ segundo a região pulmonar examinada.

\begin{tabular}{|c|c|c|c|}
\hline \multirow{2}{*}{ Região pulmonar } & \multicolumn{3}{|c|}{$\begin{array}{l}\text { Valores médios de distribuição do } \\
\text { radiofármaco* }\end{array}$} \\
\hline & 50.000 & 100.000 & 300.000 \\
\hline Cranial & $8.444^{\mathrm{a}}$ & $8.200^{\mathrm{a}}$ & $8.200^{\mathrm{a}}$ \\
\hline Média & $24.500^{\mathrm{b}}$ & $24.100^{\mathrm{b}}$ & $25.100^{\mathrm{b}}$ \\
\hline Caudal & $17.000^{\mathrm{b}}$ & $17.600^{\mathrm{b}}$ & $16.800^{\mathrm{b}}$ \\
\hline
\end{tabular}

*Médias seguidas pelas mesmas letras minúsculas não diferem entre si pelo teste de Tukey-Kramer a 5\%.
(ALVES et al., 2009; LARSON, 2009; SCHULTZ et al., 2009). Porém, como evidenciado por GREENSPAN et al. (1982) e FLÜCKINGER \& GOMEZ (1984), concordase que, em relação à perfusão pulmonar, tal técnica encontra limitações na confirmação ou exclusão de afecções relacionadas ao tromboembolismo, especialmente em estágios iniciais.

Considerando-se resultados já descritos por DANIEL et al. (2008) e DANIEL (2009), referentes ao exame cintilográfico cardíaco em cães, assim como a enormidade de protocolos prontamente estabelecidos na Medicina Humana (WU et al., 2002; MONTE et al., 2008) e a escassez destes dentro da Medicina Veterinária, este trabalho destaca a importância de uma proposta para caracterização de um padrão normal de perfusão pulmonar em cães, preliminarmente da raça Rottweiller, mediante a cintilografia com vistas à identificação lesões pulmonares, por tromboembolismo, em seus estágios iniciais, auxiliando a prática clínica, assim como a resposta do paciente ao tratamento. A vantagem do método cintilográfico sobre a radiografia convencional, em relação ao diagnóstico do tromboembolismo ou embolismo pulmonar, está na avaliação funcional dos vasos pulmonares, e a utilização do [ $\left.{ }^{99 m} \mathrm{Tc}\right](\mathrm{MAA})$ torna possível avaliar a característica vascular pulmonar quanto à presença de bloqueios do fluxo sanguíneo, que se apresenta como áreas homogêneas de cintilação ou como defeitos fotopênicos.

Embora autores como BAILE et al. (2000), GOODMAN et al. (2000) e WELLS \& RODGER, (2003) tenham demonstrado a angiografia pulmonar como "padrão ouro" no diagnóstico de lesões pulmonares, especialmente tromboembolismos, este trabalho sustenta-se nas descrições realizadas por COOPER et al. (1991) e SCHLUGER et al. (1994), quando definiram essa técnica como um método invasivo. Além disso, a literatura recente (CRONIN et al., 2008; ATTENBERGER et al., 2009) tem descrito novas técnicas diagnósticas como opção na identificação de alterações de fluxo vascular pulmonar. Desse modo, os achados cintilográficos neste trabalho tem como meta definir um padrão para cães, de modo a auxiliar, como também descrito por LETTE et al. (2003), na seleção de áreas pulmonares específicas e com maior probabilidade de aparecimento de trombos, para o emprego da angiografia, quando isso se fizer necessário.Corroborando os achados de RING et al. (1961), quando realizaram infusão de microesferas na artéria pulmonar de cães, foi verificado que as imagens obtidas após a administração do radiofármaco apresentaram uma captação adequada nos pulmões, demonstrando que o tamanho das partículas utilizadas, 
Tabela 2 - Distribuição dos valores médios da Uniformidade Integral segundo o número de partículas do radiofármaco $\left(\left[{ }^{99 \mathrm{~m}} \mathrm{Tc}\right](\mathrm{MAA})\right)$ infundidas.

\begin{tabular}{cc}
\hline № de partículas & Valores médios de UI* \\
\hline 50.000 & $17.910^{\mathrm{a}}$ \\
100.000 & $16.510^{\mathbf{b}}$ \\
300.000 & $13.060^{\mathbf{b}}$ \\
\hline
\end{tabular}

Médias seguidas pelas mesmas letras minúsculas não diferem entre si pelo teste de Tukey-Kramer a 5\%.

* Uniformidade Integral.

entre 10 e $100 \mu \mathrm{m}$, injetadas na circulação sanguínea por punção da veia cefálica, tenham sido adequadas para embolização dos vasos pulmonares de menor diâmetro.

O uso de posicionamentos e projeções variadas auxiliaram o estabelecimento de critérios de confiabilidade no padrão das imagens avaliadas, o que de modo semelhante foi também descrito por ALDERSON et al. (1978), quando pela obtenção de imagens em diferentes posicionamentos, inclusive oblíquos, puderam determinar embolia segmentar e subsegmentar em cães.

Soma-se a isso a necessidade da caracterização de imagens pulmonares cintilográficas descritas em animais normais, como o realizado neste experimento, com o intuito de sugerir um diagnóstico mais preciso sobre as inúmeras enfermidades pulmonares que ocorrem na rotina médico-veterinária, tal qual foi descrito por HOOD \& HIGHTOWER (1978) e JOHANN et al. (2005), quando discorreram sobre o padrão de alteração da perfusão associada a metástases difusas e mediastinais e à infecção por vermes cardíacos em cães, por meio da técnicas de cintilografia.

A escolha do [ $\left.{ }^{99 \mathrm{~m}} \mathrm{Tc}\right]$ como radioisótopo para a marcação do MAA dispensa qualquer preparação no animal, como, por exemplo, a administração de solução de Lugol para bloquear a captação do radiofármaco pela tireóide, como foi relatado por THRALL \& GILLETTE (1971), sendo utilizada antes da realização dos exames cintilográficos com iodo, o que facilita o procedimento com um todo.

Conforme descrito por THRALL \& GILLETTE (1971) em estudos preliminares sobre infusão de radiofármacos, neste trabalho, a administração do radiofármaco por punção venosa com uso de seringa contendo solução fisiológica evitou a injeção subcutânea da dose radioativa, evitando que o extravasamento do radiofármaco para o tecido subcutâneo comprometesse a realização do exame.
HECK \& DULEY (1974) sugeriram que o número mínimo de partículas a ser administrado para o exame da perfusão em cintilógrafo retilíneo, de um paciente humano adulto, sem que ocorra degradação da imagem periférica, seja de 60.000 microesferas e que doses acima de 150.000 partículas seriam desnecessárias. O mesmo número mínimo de partículas, ou seja, 60 partículas por grama de pulmão, foi relatado no trabalho de DWORKIN et al. (1977), que utilizaram cães à câmara de cintilação para o experimento.

No presente trabalho, embora o número de partículas de MAA tenha alterado o resultado da homogeneidade integral, na prática, a homogeneidade visual das imagens obtidas pela câmara de cintilação não foi influenciada, ainda que com a administração de um número mínimo de 50.000 partículas. De certa forma, tal resultado era esperado, uma vez que pulmões de cães Rottweiler adultos pesam aproximadamente $400 \mathrm{~g}$, que resultaria, se considerada a indicação de DWORKIN et al. (1977), em uma dosagem mínima de 24.000 partículas de MAA para obtenção de imagem uniforme. Tal homogeneidade obtida com um número crescente de partículas de MAA possivelmente constitui-se como um fator a ser considerado na avaliação diagnóstica das imagens perfusionais, quando relacionadas a pequenas lesões pulmonares.

\section{CONCLUSÃO}

Neste estudo, a cintilografia Tecnécio-99m em cães mostrou-se uma técnica segura e de fácil execução, contribuindo com informações valiosas quanto ao padrão de perfusão pulmonar visto em animais normais. A administração intravenosa do [ $\left.{ }^{99 \mathrm{~m}} \mathrm{Tc}\right]$ (MAA) foi suficiente para caracterizar adequadamente as imagens da perfusão pulmonar nas projeções dorsal, ventral, lateral direita e esquerda. Além disso, qualitativamente, o aumento dos valores da uniformidade integral, quando também aumentado o número de partículas de MAA injetadas, proporcionou maior homogeneidade da imagem cintilográfica da perfusão pulmonar, especialmente para as infusões de 300.000 , quando comparadas aos outros dois grupos de doses. Tais resultados permitem inferir um protocolo cintilográfico para avaliação da perfusão pulmonar em cães, com vistas ao auxílio à prática em Clínica Veterinária, assim como à resolução de lesões tromboembolíticas pouco sensíveis ao exame radiográfico nesses animais.

\section{COMITÊ DE ÉTICA}

Os procedimentos experimentais (protocolo n.220/ 2003) foram realizados de acordo com a Comissão de Bioética da Faculdade de Medicina Veterinária e Zootecnia da Universidade de São Paulo.

Ciência Rural, v.40, n.3, mar, 2010. 


\section{FONTES DE AQUISIÇÃO}

a - Mallinckrodt Medical B.V., Petten, Holanda

b - Siemens Ltda, Siemens, Alemanha

c - Acepran ${ }^{\circledR} 0,2 \%$ - UNIVET S/A, São Paulo, Brasil

d - Dolosal ${ }^{\circledR}$ - Cristália Produtos Químicos e Farmacêuticos Ltda, Itapira, Brasil

\section{REFERÊNCIAS}

ALDERSON, P.O. et al. Ventilation-perfusion lung imaging and selective angiography in dogs with experimental pulmonary embolism. Journal of Nuclear Medicine, v.19, n.2, p.164 171, 1978. Disponível em: <http://jnm.snmjournals.org/cgi reprint/19/2/164.pdf>. Acesso em: 22 jun. 2009.

ALVES, F.R. et al. Imagem radiográfica da cavidade torácica de cães Golden Retriever acometidos pela distrofia muscular. Pesquisa Veterinária Brasileira, v.29, p.99-104, 2009. Disponível em: <http://www.scielo.br/scielo.php?pid=S0100$736 X 2009000200002 \&$ script $=$ sci arttext\&tlng $=\mathrm{en}>$. Acesso em: 22 jun. 2009 . doi: 10.1590/S0100-736X2009000200002.

ATTENBERGER, U.I. et al. Magnetic resonance imaging of pulmonary perfusion: Technical requirements and diagnostic impact.. Radiologe, v.49, n.8, p.739-747, 2009. Disponível em: <http://dx.doi.org/10.1007/s00117-009-1880-5>. Acesso em: 22 jun. 2009. doi: 10.1007/s00117-009-1880-5.

BAILE, E.M. et al. Contrast-enhanced spiral CT is comparable to pulmonary angiography for the diagnosis of pulmonary embolism. American Journal of Respiratory and Critical Care Medicine, v.161, p.1010-1015, 2000.

BALOGH, L. et al. Veterinary nuclear medicine, scintigraphical examinations: a review. ACTA Veterinaria BRNO, v.68, n.4, p.231-239, 1999. Disponível em: <http://vfu-www.vfu.cz/ acta-vet/vol68/pdf/68_231.pdf $>$. Acesso em: 22 jun. 2009.

BOOKSTEIN, J.J. et al. Diagnosis of pulmonary embolism: experimental evaluation of the accuracy of scintigraphically guided pulmonary arteriography. Radiology, v.136, n.1, p.1523, 1980. Disponível em: <http://radiology.rsna.org/content/ 136/1/15.full.pdf + html>. Acesso em: 22 jun. 2009

BUCKLEY, S.E. et al. Gamma camera scintigraphy of tumours using ${ }^{195 \mathrm{~m} P t-c i s p l a t i n . ~ P h y s i c s ~ i n ~ M e d i c i n e ~ B i o l o g y, ~ v .51, ~}$ p.1325-1332, 2006. Disponível em: < http://dx.doi.org/ 10.1088/0031-9155/51/5/020>. Acesso em: 22 jun. 2009. doi: $10.1088 / 0031-9155 / 51 / 5 / 020$.

CANECA, O.A.F. et al. Alterações pulmonares cintilográficas e espirométricas em pacientes jovens portadores de esquistossomose mansônica tratados clínica e cirurgicamente na infância. Revista do Colégio Brasileiro de Cirurgia, v.29, n.5, p.267-270, 2002. Disponível em: <http:// w w w. s c i e 1 o.br/s c i e 1 o.p h p ? p id = S 0100 $69912002000500004 \&$ script $=$ sci arttext $>$. Acesso em: 22 jun. 2009. doi: 10.1590/S0100-69912002000500004

COOPER, T.J. et al. Survery on the use of pulmonary scintigraphy and angiography for supspected pulmonary thromboembolism in the UK. Clinical Radiology, v.43, p.243-245, 1991. Disponível em: < http://dx.doi.org/ 10.1016/ S0009-9260(05)80246-6>. Acesso em: 22 jun. 2009. doi:10.1016/S0009-9260(05)80246-6.
CRONIN, P. et al. The role of multidetector computed tomography angiography for the diagnosis of pulmonary embolism. Seminars in Nuclear Medicine, v.38, n.6, p.418431, 2008. Disponível em: <http://dx.doi.org/10.1053/ j.semnuclmed.2008.07.002 >. Acesso em: 22 jun. 2009. doi:10.1053/j.semnuclmed.2008.07.002.

DANIEL, G.B. et al. Per rectal portal scintigraphy using ${ }^{99 m}$ Technetium pertechnetate to diagnose portosystemic shunts in dogs and cats. Journal of Veterinary Internal Medicine, v.5, n.1, p.23-27, 2008. Disponível em: <http://dx.doi.org/ 10.1111/j.1939-1676.1991.tb00926.x>. Acesso em: 22 jun. 2009. doi:10.1111/j.1939-1676.1991.tb00926.x.

DANIEL, G.B. Scintigraphic diagnosis of portosystemic shunts. Veterinary Clinics of North America: Small Animal Practice, v.39, p.793-810, 2009. Disponível em: <http:// dx.doi.org/ doi:10.1016/j.cvsm.2009.04.009>. Acesso em: 22 jun. 2009. doi:10.1016/j.cvsm.2009.04.009.

DWORKIN, H.J. et al. Effect of particle number on lung perfusion images. Journal of Nuclear Medicine, v.18, n.3 p.260-262, 1977.

FLÜCKINGER, M.A.; GOMEZ, J.A. Radiographic findings in dog with spontaneous pulmonary thrombosis or embolism. Veterinary Radiology, v.25, n.3. p.124-131, 1984. Disponível em: <http://dx.doi.org/ 10.1111/j.17408261.1984.tb01923.x>. Acesso em: 22 jun. 2009. doi: 10.1111/j.1740-8261.1984.tb01923.x

GOODMAN, L.R. et al. Subsequent pulmonary embolism: risk after a negative helical CT pulmonary angiogram - prospective comparison with scintigraphy. Radiology, v.215, p.535-542, 2000. Disponível em: <http://radiology.rsna.org/content/215/ 2/535.full.pdf+html>. Acesso em: 22 jun. 2009.

GREENSPAN, R.H. et al. Accuracy of the chest radiograph in diagnosis of pulmonary embolism. Investigative Radiology, v.17, n.6, p.539-543, 1982

HARNAGEL, S.H. et al. The use of ${ }^{99 \mathrm{~m}} \mathrm{Tc}$ radioaerosol ventilation and macroaggregated albumin perfusion imaging for the detection of pulmonary emboli in the dog. Veterinary Radiology, v.30, n.1, p.22-27, 1989. Disponível em: <http:/ /dx.doi.org/10.1111/j.1740-8261.1989.tb00748.x>. Acesso em: 22 jun. 2009. doi: 10.1111/j.1740-8261.1989.tb00748.x.

HECK, L.L.; DULEY, J.W. Statistical considerations in lung imaging with ${ }^{99 \mathrm{~m}} \mathrm{Tc}$ albumin particles. Radiology, v.113, n.3, p.673-679, 1974

HOOD, D.M.; HIGHTOWER, D. Clinical pulmonary perfusion imaging in the dog. American Journal of Veterinary Research, v.39, n.11, p.1794-1798, 1978.

JOHANN, L. et al. Sensitivity of radiographic detection of lung metastases in the dog. Veterinary Radiology and Ultrasound, v.27, n.3, p.74-78, 2005. Disponível em: <http:/ /dx.doi.org/10.1111/j.1740-8261.1986.tb00006.x>. Acesso em: 22 jun. 2009. doi:10.1111/j.1740-8261.1986.tb00006.x.

LARSON, M.M. Ultrasound of the thorax (noncardiac). Veterinary Clinic North America: Small Animal Practices, v.39, n.4, p.733-745, 2009. Disponével em: <http:/ /dx.doi.org/10.1016/j.cvsm.2009.04.006 >. Acesso em: 22 jun. 2009. doi:10.1016/j.cvsm.2009.04.006. 
LETTE, J. et al. Scintigraphic probability and angiographic diagnostic certainty in acute pulmonary embolism. Clinical Nuclear Medicine, v.28, n.11, p.897-904, 2003.

MARTIN, B.W. Distribution of small animal heart diseases in São Paulo-Brasil. Veterinary Radiology and Ultrasound, v.44, p.233, 2003.

MARTIN, B.W. et al. Experimental evaluation of brain spect using D.L.HMPAO-99MTC in dogs - anatomo phisiological implications. Brazilian Journal of Veterinary Research and Animal Science, v.32, p.1-4, 1995.

MONTE, G.U. et al. Ressonância magnética vs cintilografia com pirofosfato marcado com tecnécio-99m para a detecção de necrose miocárdica perioperatória. Arquivo Brasileiro de Cardiologia, v.91, n.2, p.126-131, 2008. Disponível em: <http://dx.doi.org/10.1590/S0066-782X2008001400009>. Acesso em: 22 jun. 2009. doi: 10.1590/S0066782 X2008001400009.

$\mathrm{OCH}$, J.G. et al. Acceptance testing of magnetic resonance imaging systems: report of AAPM Nuclear Magnetic Resonance task group n.6. Med Physics, v.19, p.217-229, 1992. Disponível em: <http://www.aapm.org/pubs/reports/ RPT 34.pdf>. Acesso em: 22 jun. 2009

OSBORNE, D.R. et al. Detection of pulmonary emboli in dogs: comparison on single photon emission computed tomography, gamma camera imaging, and angiography. Radiology, v.146, n.2, p.493-497, 1983 .

PRICE, R.R. et al. Quality assurance methods and phantoms for magnetic resonance imaging: report of AAPM Nuclear Magnetic Resonance task group n.1. Med Physics, v.17, p.287-295, 1990. Disponível em: <http://www.aapm.org/pubs/ reports/rpt_28.pdf>. Acesso em: 22 jun. 2009.

RING, G.C. et al. Size of microspheres passing through pulmonary circuit in the dog. American Journal of Physiology, v.200, p.1191-1196, 1961.

RHODES, B.A. et al. Lung Scanning with ${ }^{99 \mathrm{~m}} \mathrm{Tc}-\mathrm{microspheres.}$ Radiology, v.99, n.3, p.613-621, 1971.

SARIERLER, M. et al. Scintigraphic assessment of hepatobiliary functions in healthy adult dogs. Turkish Journal of Veterinary and Animal Sciences, v.29, p.1001-1006, 2005. Disponível em: <http://journals.tubitak.gov.tr/veterinary/ issues/vet-05-29-4/vet-29-4-9-0403-11.pdf>. Acesso em: 22 jun. 2009.

SCHLUGER, N. et al. Diagnosis of pulmonary embolism at a large teaching hospital. Journal of Thoracic Imaging, v.9, p.180-184, 1994.

SCHULTZ, R.M. et al. Radiography, computed tomography and virtual bronchoscopy in four dogs and two cats with lung lobe torsion. Journal of Small Animal Practices, v.50, n.7, p.360-363, 2009. Disponível em: <http://dx.doi.org/ 10.1111/j.1748-5827.2009.00728.x>. Acesso em: 22 jun. 2009. doi: 10.1111/j.1748-5827.2009.00728.x.

SCHWARZ, T. et al. Bone scintigraphy in the investigation of occult lameness in the dog. Journal of Small Animal Practices, v.45 n.5, p.232-237, 2004. Disponível em: <http:/ /dx.doi.org/10.1111/j.1748-5827.2004.tb00228.x >. Acesso em: 22 jun. 2009. doi: 10.1111/j.1748-5827.2004.tb00228.x.

SUGERMAN, H.J. et al. Sensitivity of scintigraphy for detection of pulmonary capillary albumin leak in canine oleic acid ARDS. J Trauma, v.21, n.7, p.520-527, 1981.

THRALL D.E. et al. Scintigraphic evaluation of pulmonary perfusion in dogs experimentally infected with Dirofilaria immitis. American Journal Veterinary Research, v.40, n.10, p.1426-1432, 1979.

THRALL, D.E.; GILlETTE, E.L. Canine lung scanning. Journal of American Veterinary Radiology Society, v.12, p.88-95, 1971 .

TIDWELL, A.S. Diagnosis pulmonary imaging. Problems in Veterinary Medicine, v.4, n.2, p.239-264, 1992.

WELLS, P.S.; RODGER, M. Diagnosis of pulmonary embolism: when is imaging needed? Clinics Chest Medicine, v.24. p.13-28, 2003. Disponível em: <http://dx.doi.org/10.1016/ S0272-5231(02)00052-7>. Acesso em: 22 jun. 2009. doi:10.1016/S0272-5231(02)00052-7.

WU, M.T. et al. Prediction of postoperative lung function in patients with lung cancer: comparison of quantitative CT with perfusion scintigraphy. American Journal of Roentgenology, v.178, n.3, p.667-672, 2002. Disponível em: <http://www.ajronline.org/cgi/reprint/178/3/667>. Acesso em: 22 jun. 2009. 